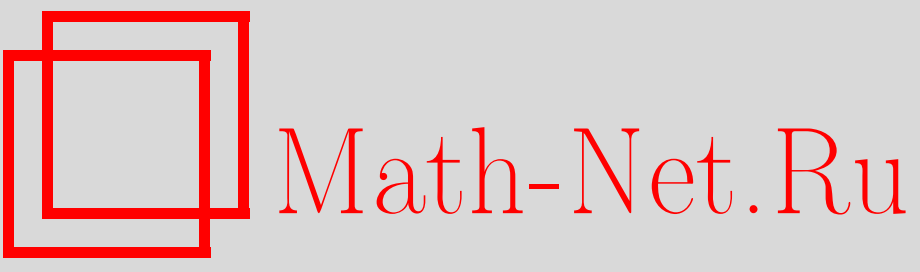

О. И. Богоявленский, Кольцо когомологий для гладких отображений, УМН, 1999, том 54, выпуск 1, 11-20

DOI: https://doi.org/10.4213/rm115

Использование Общероссийского математического портала Math-Net.Ru подразумевает, что вы прочитали и согласны с пользовательским соглашением

http://www.mathnet.ru/rus/agreement

Параметры загрузки:

IP : 54.224 .60 .19

26 апреля 2023 г., 15:09:12 


\title{
КОЛЬЦО КОГОМОЛОГИЙ ДЛЯ ГЛАДКИХ ОТОБРАЖЕНИЙ
}

\author{
О. И. БогОявлЕнСКий
}

\section{СОДЕРЖАНИЕ}

$\S 1 . A-B-C$-когомологии для динамических систем

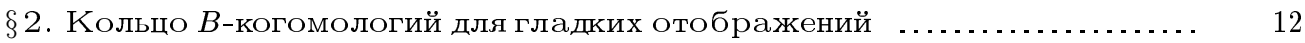

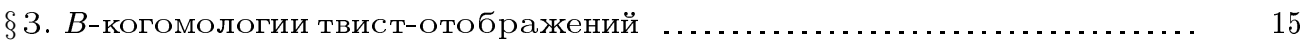

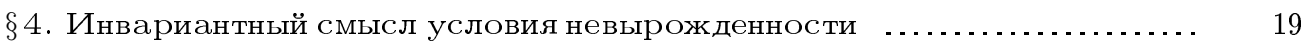

Список литературы .............................................. 20

\section{$\S$ 1. $A-B-C$-когомологии для динамических систем}

I. Напомним конструкцию $A-B$ - $C$-когомологий динамических систем, введенных в наших работах [3]-[6]. Пусть $\Lambda_{V}^{m}$ - линейное пространство гладких дифференциальных $m$-форм на многообразии $M^{n}$, которые являются инвариантными по отношению к некоторой гладкой динамической системе $V$

$$
\dot{x}^{i}=V^{i}\left(x^{1}, \ldots, x^{n}\right)
$$

на многообразии $M^{n}$. Оператор внутреннего произведения $i_{V}$ и оператор внешнего дифференщирования $d$ действуют на $V$-инвариантных дифференциальных формах. Любая $V$-инвариантная $m$-форма $\omega_{m}$ удовлетворяет уравнению $L_{V} \omega_{m}=0$, где $L_{V}=i_{V} \circ d+d \circ i_{V}$ есть оператор Ли-дифференцирования. Поэтому операторы $i_{V}$ и $d$ удовлетворяют уравнениям

$$
i_{V}^{2}=0, \quad \mathrm{~d}^{2}=0, \quad i_{V} \circ \mathrm{d}=-\mathrm{d} \circ i_{V}
$$

на линейном пространстве $V$-инвариантных дифференциальных форм. Вследствие уравнений (1.2) оператор $d_{V}=i_{V} \circ d=-d \circ i_{V}$ удовлетворяет уравнениям

$$
\mathrm{d}_{V}^{2}=0, \quad \mathrm{~d}_{V} \circ i_{V}=i_{V} \circ \mathrm{d}_{V}=0, \quad \mathrm{~d}_{V} \circ \mathrm{d}=\mathrm{d} \circ \mathrm{d}_{V}=0 .
$$

Линейные пространства $\Lambda_{V}^{m} V$-инвариантных дифференщиальных $m$-форм с тремя операторами $i_{V}, d$ и $d_{V}=i_{V}$ ○ $d$ образуют $A$-, $B$ - и $C$-комплексы соответственно

$$
\begin{array}{ll}
A: & 0 \longleftarrow \Lambda_{f}^{0} \stackrel{i_{V}}{\longleftarrow} \Lambda_{f}^{1} \stackrel{i_{V}}{\longleftarrow} \ldots \stackrel{i_{V}}{\longleftarrow} \Lambda_{f}^{n-1} \stackrel{i_{V}}{\longleftarrow} \Lambda_{f}^{n} \longleftarrow 0 \\
B: & 0 \longrightarrow \Lambda_{f}^{0} \stackrel{d}{\longrightarrow} \Lambda_{f}^{1} \stackrel{d}{\longrightarrow} \ldots \stackrel{d}{\longrightarrow} \Lambda_{f}^{n-1} \stackrel{d}{\longrightarrow} \Lambda_{f}^{n} \longrightarrow 0 \\
C: & \stackrel{d_{V}}{\longrightarrow} \Lambda_{f}^{m} \stackrel{d_{V}}{\longrightarrow} \Lambda_{f}^{m} \stackrel{d_{V}}{\longrightarrow} \Lambda_{f}^{m} \stackrel{d_{V}}{\longrightarrow} .
\end{array}
$$


II. Линейные пространства $H_{A}^{*}\left(V, M^{n}\right), H_{B}^{*}\left(V, M^{n}\right)$ и $H_{C}^{*}\left(V, M^{n}\right)$ являются когомологиями по отношению к операторам $i_{V}, d$ и $d_{V}$ соответственно:

$$
\begin{aligned}
H_{A}^{*}\left(V, M^{n}\right)= & \operatorname{Ker} i_{V} / \operatorname{Im} i_{V}, \quad H_{B}^{*}\left(V, M^{n}\right)=\operatorname{Ker} \mathrm{d} / \operatorname{Imd}, \\
& H_{C}^{*}\left(V, M^{n}\right)=\operatorname{Ker} \mathrm{d}_{V} / \operatorname{Imd}_{V} .
\end{aligned}
$$

Фактор-пространства $H_{A}^{*}\left(V, M^{n}\right)$ и $H_{B}^{*}\left(V, M^{n}\right)(1.4)$ наследуют кольцевую структуру, определенную внешним произведением $V$-инвариантных дифференциальных форм, поскольку операторы $i_{V}$ и $d$ являются косыми дифференцированиями. Оператор $d_{V}=i_{V} \circ d$ не является дифференцированием. Поэтому линейные пространства $H_{C}^{*}\left(V, M^{n}\right)$ имеют только групповую структуру.

ЗАмЕчАниЕ $1 . A$-когомологии $H_{A}^{*}\left(V, M^{n}\right)$ образуют дифференциальный комплекс по отношению к оператору $d$. $B$-когомологии $H_{B}^{*}\left(V, M^{n}\right)$ образуют дифференциальный комплекс по отношению к оператору $i_{V}$. $C$-когомологии $H_{C}^{*}\left(V, M^{n}\right)$ образуют два дифференциальных комплекса по отношению к операторам $i_{V}$ и $d$. Когомологии всех этих дифференциальных комплексов являются дополнительными инвариантами динамической системы $V$.

\section{$\S$ 2. Кольцо $B$-когомологий для гладких отображений}

I. В предыдушем параграфе мы ввели $A-B-C$-когомологии для динамических систем. Среди них только $B$-когомологии имеют аналоги для дискретных динамических систем. Пусть $f$-некотороегладкое отображение многообразия $M^{n}$ в себя. Пусть $\Lambda_{f}^{q}$ означает линейное пространство $f$-инвариантных $k$-форм $\omega_{q}: f^{*} \omega_{q}=\omega_{q}$. Рассмотрим дифференциальньй комплекс $f$-инвариантных дифференциальных форм

$$
0 \longrightarrow \Lambda_{f}^{0} \stackrel{d}{\longrightarrow} \Lambda_{f}^{1} \stackrel{d}{\longrightarrow} \ldots \stackrel{d}{\longrightarrow} \Lambda_{f}^{n-1} \stackrel{d}{\longrightarrow} \Lambda_{f}^{n} \longrightarrow 0
$$

Мы определяем $B$-когомологии гладкого отображения $f$ формулой

$$
H_{B}^{*}\left(f, M^{n}\right)=\operatorname{Ker} \mathrm{d} / \operatorname{Im} \mathrm{d} .
$$

Внешнее произведение $f$-инвариантных дифференциальных форм индуцирует кольцевую структуру в когомологиях $H_{B}^{*}\left(f, M^{n}\right)$.

Пусть диффеоморфизм $f_{\tau}$ является сдвигом на время $\tau$ вдоль траекторий динамической системы $V$. В этом случае определен кольцевой гомоморфизм

$$
H_{B}^{*}\left(V, M^{n}\right) \rightarrow H_{B}^{*}\left(f_{\tau}, M^{n}\right) .
$$

Для многих динамических систем $V$ этот гомоморфизм становится изоморфизмом при $\tau \rightarrow 0$.

ЗАмЕчаниЕ 2. Представленные конструкции показьвают, что $B$-когомологии гладких отображений $f$ и $A$ - $B$ - $C$-когомологии динамических систем $V$ отличаются от когомологического уравнения $R(f(x))-R(x)=G(x)$, которое было введено Гильбертом [10] и исследовано Колмогоровым [12], см. также [11]. 
ЗАмечАниЕ 3. В работе [6] мы показали, что любая группа $G$ симметрий динамической системы $V$ обладает линейными представлениями в $A-B$ - $C$-когомологиях. Аналогично, любая группа $\Gamma$ диффеоморфизмов многообразия $M^{n}$, которые коммутируют с гладким отображением $f: M^{n} \rightarrow M^{n}$, имеет линейные представления в когомологиях $H_{B}^{m}\left(f, M^{n}\right), m=1, \ldots, n$.

II. Существует кольцевой гомоморфизм

$$
\alpha: H_{B}^{*}\left(f, M^{n}\right) \rightarrow H^{*}\left(M^{n}\right),
$$

который преобразует $B$-когомологический класс $f$-инвариантных замкнутых $q$-форм в соответствуюший класс когомологий де-Рама общих замкнутых $q$-форм.

Гомоморфизм $\alpha$ имеет обратньй и поэтому является изоморфизмом для следуюших диффеоморфизмов $f$ :

1) Предположим, что диффеоморфизм $f: M^{n} \rightarrow M^{n}$ гомотопен тождественному отображению и является периодическим с периодом $p$. Отображения

$$
f^{m}: M^{n} \rightarrow M^{n}, \quad f^{p}=\mathrm{id}
$$

определяют действие циклической групшы $\mathbb{Z}_{p}$. Для любой замкнутой $q$-формы $\omega_{q}$ мы определяем $q$-форму

$$
\alpha^{-1} \omega_{q}=\frac{1}{p} \sum_{m=1}^{p}\left(f^{m}\right)^{*}\left(\omega_{q}\right) .
$$

Очевидно, что $q$-форма $\alpha^{-1} \omega_{q}$ является замкнутой и инвариантной по отношению к диффеоморфизму $f ; q$-форма $\alpha^{-1} \omega_{q}$ принадлежит тому же классу когомологий де-Рама в $H^{q}\left(M^{n}\right)$, что и замкнутая $q$-форма $\omega_{q}$, поскольку $q$-формы $\left(f^{m}\right)^{*}\left(\omega_{q}\right)$ когомологичны $\omega_{q}$ при всех $m$, так как отображение $f$ гомотопно тождественному отображению. Поэтому $\alpha \circ \alpha^{-1}=\mathrm{id}$ в $H^{q}\left(M^{n}\right)$ и, следовательно, отображение $\alpha$ является изоморфизмом.

2) Пусть $M^{n}=X^{n-k} \times \mathbb{T}^{k}$, где $X^{n-k}$-некоторое гладкое $(n-k)$-мерное многообразие с атласом локальных координат $x^{1}, \ldots, x^{n}$ и $\mathbb{T}^{k}$ есть $k$-мерньй тор с угловыми координатами $\varphi_{1}, \ldots, \varphi_{k}$. Рассмотрим диффеоморфизм

$$
f:\left(x^{i}, \varphi_{j}\right) \rightarrow\left(x^{i}, \varphi_{j}+a_{j}\right)
$$

многообразия $M^{n}$ в себя. Здесь $a_{1}, \ldots, a_{k}-$ произвольные рационально независимые постоянные.

Пусть $g(x, \varphi)$ - произвольная гладкая функция на $M^{n}$. Диффеоморфизм $f(2.2)$ тора $\mathbb{T}^{k}$ в себя является эргодическим, если константы $a_{1}, \ldots, a_{k}$ рационально независимы [1], [2], [9]. Отсюда мы получаем

$$
\overline{g(x)}=\lim _{N \rightarrow \infty} \frac{1}{N} \sum_{m=1}^{N} g\left(x^{i}, \varphi_{j}+m a_{j}\right)=\frac{1}{(2 \pi)^{k}} \int_{\mathbb{T}^{k}} g(x, \varphi) \mathrm{d} \varphi_{1} \wedge \cdots \wedge \mathrm{d} \varphi_{k} .
$$

Пусть $\omega_{q}-$ произвольная замкнутая $q$-форма

$$
\omega_{q}=\sum_{\ell+m=q} \sum_{i, j} a_{i, j}(x, \varphi) \mathrm{d} x^{i_{1}} \wedge \cdots \wedge \mathrm{d} x^{i_{\ell}} \wedge \mathrm{d} \varphi_{j_{1}} \wedge \cdots \wedge \mathrm{d} \varphi_{j_{m}}
$$

на многообразии $M^{n}$. Для всех диффеоморфизмов

$$
f^{m}:\left(x^{i}, \varphi_{j}\right) \rightarrow\left(x^{i}, \varphi_{j}+m a_{j}\right),
$$


дифференциалы $d\left(f^{m}\right)$ являются тождественными отображениями касательных пространств $T_{(x, \varphi)}\left(X^{n-k} \times \mathbb{T}^{k}\right)$. Используя этот факт, мы получаем равенство

$$
\lim _{N \rightarrow \infty} \frac{1}{N} \sum_{m=1}^{N}\left(f^{m}\right)^{*}\left(\omega_{q}\right)=\sum_{\ell+m=q} \sum_{i, j} \overline{a_{i, j}(x)} \mathrm{d} x^{i_{1}} \wedge \cdots \wedge \mathrm{d} x^{i_{\ell}} \wedge \mathrm{d} \varphi_{j_{1}} \wedge \cdots \wedge \mathrm{d} \varphi_{j_{m}}
$$

Мы определяем

$$
\alpha^{-1} \omega_{q}=\bar{\omega}_{q}=\lim _{N \rightarrow \infty} \frac{1}{N} \sum_{m=1}^{N}\left(f^{m}\right)^{*}\left(\omega_{q}\right)
$$

Очевидно, что $q$-форма $\bar{\omega}_{q}$ является замкнутой и инвариантной по отношению к диффеоморфизму (2.2). Очевидно, что $\alpha^{-1} \circ \alpha=$ id. Построенная $f$-инвариантная замкнутая $q$-форма $\bar{\omega}_{q}$ принадлежит тому же классу когомологий де-Рама в $H^{q}\left(M^{n}\right)$, что и замкнутая $q$-форома $\omega_{q}$. Действительно, $q$-форомы $\left(f^{m}\right)^{*}\left(\omega_{q}\right)$ когомологичны $\omega_{q}$ при всех $m$, поскольку диффеоморфизм $f(2.2)$ гомотопен тождественному отображению. Поэтому $\alpha \circ \alpha^{-1}=\mathrm{id}$ в $H^{q}\left(M^{n}\right)$ и, следовательно, отображение $\alpha$ является изоморфизмом. Таким образом, мы доказали, что отображение $\alpha$ является изоморфизмом двух колец когомологий

$$
\alpha: H_{B}^{*}\left(f, M^{n}\right)=H^{*}\left(M^{n}\right)
$$

для диффеоморфизмов $f(2.2)$ многообразий $M^{n}=X^{n-k} \times \mathbb{T}^{k}$ в себя.

III. Пусть $(n+1)$-мерное многообразие $S_{f} M^{n}$ является надстройкой [11] многообразия $M^{n}$, соответствуюшей некоторому диффеоморфизму $f: M^{n} \rightarrow M^{n}$. Пусть $t \in[0,1]$ - "вертикальная" координата на $S_{f} M^{n}$, которая определяет векторное поле $V=\partial / \partial t$. Отображение $f$ индуцирует диффеоморфизм $f_{1}$ надстройки $S_{f} M^{n}$ : $f_{1}(x, t)=(f(x), t)$. Очевидно, что диффеоморфизм $f_{1}$ является сдвигом на единицу времени вдоль траекторий векторного поля $V=\partial / \partial t$ на многообразии $S_{f} M^{n}$.

Любая $f$-инвариантная $k$-форма $\omega_{k}$ на $M^{n}$ индуцирует $V$-инвариантную $k$-форму $E \omega_{k}$ на $S_{f} M^{n}$, которая определена уравнениями $E_{t}^{*}\left(E \omega_{k}\right)=\omega_{k}, i_{V} E \omega_{k}=0$, где отображения $E_{t}$ являются вложениями $E_{t}: M^{n} \rightarrow S_{f} M^{n}$, где $E_{t}(x)=(x, t)$.

Теорема 1. Для любого диффеоморфизма $f: M^{n} \rightarrow M^{n}$ следующие два кольиа когомологий изоморфнь:

$$
H_{B}^{*}\left(V, S_{f} M^{n}\right)=H_{B}^{*}\left(f, M^{n}\right) \otimes H^{*}\left(S^{1}\right) .
$$

Здесь $H^{*}\left(S^{1}\right)=\mathbb{R}^{1} \oplus \mathbb{R}^{1}$ есть кольцо когомологий де-Рама окружности $S^{1}$.

ДоказАТЕльство. Пусть $\eta_{k+1}$ - некоторая $V$-инвариантная $(k+1)$-форма на $S_{f} M^{n}$. $V$-инвариантность означает, что форма $\eta_{k+1}$ переходит в себя при сдвиге $f_{\tau}: t \rightarrow t+\tau$ для любого $\tau$. Поэтому $(k+1)$-форма $\eta_{k+1}$ имеет вид

$$
\eta_{k+1}=\mathrm{d} t \wedge E \omega_{k}+E \omega_{k+1},
$$

в открытом цилиндре $I \times M^{n}, 0<t<1$. Здесь $\omega_{k}$ и $\omega_{k+1}$ - некоторые $k$ - и $(k+1)$-дифференциальные формы на $M^{n}$. После сдвига $f_{1} V$-инвариантная $(k+1)$-форма $\eta_{k+1}$ преобразуется в себя:

$$
f_{1}^{*} \eta_{k+1}=\mathrm{d} t \wedge E f^{*} \omega_{k}+E f^{*} \omega_{k+1}=\eta_{k+1} .
$$

Отсюда мы получаем $f^{*} \omega_{k}=\omega_{k}, f^{*} \omega_{k+1}=\omega_{k+1}$. 
Обратно, если две дифференциальные формы $\omega_{k}$ и $\omega_{k+1}$ на $M^{n}$ являются $f$-инвариантными, то $(k+1)$-форма $\eta_{k+1}(2.5)$ корректно определена на многообразии $S_{f} M^{n}$ и $V$-инвариантна. Форма $\eta_{k+1}(2.5)$ замкнута или точна тогда и только тогда, когда формы $\omega_{k}$ и $\omega_{k+1}$ замкнуты или точны. Это заканчивает доказательство изоморфизма (2.4).

IV. Пусть $M^{2 k}$ - некоторое симплектическое многообразие с симплектической структурой $\omega$, и пусть $f: M^{2 k} \rightarrow M^{2 k}$ - эргодический диффеоморфизм общего положения, сохраняющий симплектическую структуру $\omega$. Кольцо $B$-когомологий для такого диффеоморфизма $f$ общего положения изоморфно фактор-кольцу полиномов от одной переменной $u$ :

$$
H_{B}^{*}\left(f, M^{2 k}\right)=\mathbb{R}[u] / u^{k+1} \mathbb{R}[u] .
$$

Образующая $u \in H_{B}^{2}\left(f, M^{2 k}\right)$ соответствует $f$-инвариантной симплектической структуре $\omega$. Отметим, что рассмотренные ранее диффеоморфизмы (2.2) не являются эргодическими. Поэтому соответствуюшие кольца $B$-когомологий $(2.3)$ неизоморфны кольцам $B$-когомологий (2.6).

ЗАмЕчАНИЕ 4. Изоморфизм (2.3) для “интегрируемых” диффеоморфизмов (2.2) и изоморфизм (2.6) для "неинтегрируемых" эргодических диффеоморфизмов $f$ общего положения показывают, что кольцо когомологий $H_{B}^{*}\left(f, M^{n}\right)$ является новым инвариантом, которьй одновременно характеризует топологические свойства многообразия $M^{n}$ и глобальные свойства гладкого отображения $f: M^{n} \rightarrow M^{n}$.

\section{$\S$ 3. $B$-когомологии твист-отображений}

Пусть многообразие $M^{2 k}$ является тороидальной областью $\mathscr{O}=B_{a} \times \mathbb{T}^{k}$, где $B_{a} \subset \mathbb{R}^{k}-$ шар

$$
B_{a}: \sum_{j=1}^{k}\left(I_{j}-I_{j 0}\right)^{2}<a^{2},
$$

и $\mathbb{T}^{k}$ - тор с угловыми координатами $\varphi_{1}, \ldots, \varphi_{k}$. Определим диффеоморфизм $f: \mathscr{O} \rightarrow \mathscr{O}$ формулой

$$
f\left(I_{j}, \varphi_{j}\right)=\left(I_{j}, \varphi_{j}+\alpha_{j}(I)\right), \quad j=1, \ldots, k,
$$

где $\alpha_{j}(I)$ являются произвольными гладкими функциями. Диффеоморфизм (3.1) называется твист-отображением и является отображением Пуанкаре для интегрируемых гамильтоновых систем в тороидальных областях на подмногообразиях постоянной энергии. Диффеоморфизмы (3.1) возникают также в виде дискретизаций интегрируемых гамильтоновых систем.

ТЕОРема 2. Предположим, что твист-отображение $f(3.1)$ nри $k \geqslant 2$ удовлетворяет следующему условию невырожденности:

$$
\operatorname{det}\left\|\frac{\partial \alpha_{\ell}(I)}{\partial I_{j}}\right\| \neq 0, \quad \ell, j=1, \ldots, k,
$$

и что отображсене $\left(I_{1}, \ldots, I_{k}\right) \rightarrow\left(\alpha_{1}(I), \ldots, \alpha_{k}(I)\right)$ является взаимно однозначным в иаре $B_{a}$. Тогда первые пять В-когомологических групп имеют вид

$$
H_{B}^{0}(f, \mathscr{O})=\mathbb{R}^{1}, \quad H_{B}^{1}(f, \mathscr{O})=0, H_{B}^{2}(f, \mathscr{O})=\mathbb{R}^{\infty}, H_{B}^{3}(f, \mathscr{O})=0, \quad H_{B}^{4}(f, \mathscr{O})=\mathbb{R}^{\infty} .
$$


ДокАЗАТЕльство. Условие невырожденности (3.2) означает, что функции $J_{\ell}=$ $\alpha_{\ell}(I)$ образуют новую систему координат в шаре $B_{a}$. Диффеоморфизм $f(3.1)$ принимает вид $f\left(J_{i}, \varphi_{i}\right)=\left(J_{i}, \varphi_{i}+J_{i}\right)$ в новых координатах.

Уравнение инвариантности $f^{*} \theta=\theta$ для дифференциальной 1-формы

$$
\theta=\theta_{i}(J, \varphi) \mathrm{d} J_{i}+\theta_{k+i}(J, \varphi) \mathrm{d} \varphi_{i}
$$

эквивалентно системе уравнений

$$
\begin{gathered}
\theta_{k+i}(J, \varphi)=\theta_{k+i}(J, \varphi+J), \\
\theta_{i}(J, \varphi)=\theta_{i}(J, \varphi+J)+\theta_{k+i}(J, \varphi+J) .
\end{gathered}
$$

Итерируя уравнение (3.3) $N$ раз, мы получаем уравнение

$$
\theta_{k+i}(J, \varphi)=\theta_{k+i}(J, \varphi+N J)
$$

для произвольного целого числа $N$. Для общего вектора $J=\left(J_{1}, \ldots, J_{k}\right)$ точки $\varphi+N J$ всюду плотны в торе $\mathbb{T}^{k}$. Функции $\theta_{k+i}(J, \varphi)$ являются гладкими. Поэтому уравнения (3.5) влекут, что функции $\theta_{k+i}(J, \varphi)$ зависят только от переменных $J_{i}: \theta_{k+i}(J, \varphi)=\theta_{k+i}(J)$.

Итерируя уравнение (3.4) $N$ раз, мы получаем

$$
\theta_{i}(J, \varphi)=\theta_{i}(J, \varphi+N J)+N \theta_{k+i}(J)
$$

для произвольного целого числа $N$. Гладкие функции $\theta_{i}(J, \varphi)$ ограничены по модулю на любом торе $\mathbb{T}^{k}$. Правая часть уравнения (3.6) ограничена при всех $N$, только если $\theta_{k+i}(J)=0$. Поэтому уравнение (3.6) влечет $\theta_{i}(J, \varphi)=\theta_{i}(J)$ по тем же причинам, что и для уравнения (3.5). Таким образом, мы доказали, что любая $f$-инвариантная 1-форма $\theta$ имеет вид

$$
\theta=\theta_{i}(J) \mathrm{d} J_{i}
$$

Если 1-форма $\theta$ (3.7) является замкнутой, $d \theta=0$, то, применяя лемму Пуанкаре, мы получаем $\theta=d F(J)$, где $F(J)$ - некоторая гладкая функция. Поэтому первая когомологическая группа $H_{B}^{1}(f, \mathscr{O})$ равна нулю.

2а) Уравнение инвариантности $f^{*} \omega=\omega$ для дифференциальной 2-формы

$$
\omega=a_{\ell i}(J, \varphi) \mathrm{d} J_{\ell} \wedge \mathrm{d} J_{i}+b_{\ell i}(J, \varphi) \mathrm{d} J_{\ell} \wedge \mathrm{d} \varphi_{i}+c_{\ell i}(J, \varphi) \mathrm{d} \varphi_{\ell} \wedge \mathrm{d} \varphi_{i}
$$

эквивалентно системе уравнений

$$
\begin{aligned}
& c_{\ell i}(J, \varphi)=c_{\ell i}(J, \varphi+J) \\
& b_{\ell i}(J, \varphi)=b_{\ell i}(J, \varphi+J)+2 c_{\ell i}(J, \varphi+J), \\
& a_{\ell i}(J, \varphi)=a_{\ell i}(J, \varphi+J)+\frac{1}{2}\left(b_{\ell i}(J, \varphi+J)-b_{i \ell}(J, \varphi+J)\right)+c_{\ell i}(J, \varphi+J)
\end{aligned}
$$

Уравнения (3.8) и (3.9) имеют тот же вид, что и уравнения (3.3) и (3.4). Поэтому, применяя те же аргументы, мы получаем $b_{\ell i}(J, \varphi)=b_{\ell i}(J), c_{\ell i}(J, \varphi)=0$. Подставляя эти формулы и итерируя уравнение (3.10) $N$ раз, мы выводим

$$
a_{\ell i}(J, \varphi)=a_{\ell i}(J, \varphi+N J)+\frac{N}{2}\left(b_{\ell i}(J)-b_{i \ell}(J)\right) .
$$


Это уравнение имеет тот же вид, что и уравнение (3.6). Поэтому те же аргументы доказывают, что $a_{\ell i}(J, \varphi)=a_{\ell i}(J), b_{\ell i}(J)-b_{i \ell}(J)=0$. Таким образом, мы установили, что любая $f$-инвариантная 2-форма $\omega$ имеет вид

$$
\omega=a_{\ell i}(J) \mathrm{d} J_{\ell} \wedge \mathrm{d} J_{i}+b_{\ell i}(J) \mathrm{d} J_{\ell} \wedge \mathrm{d} \varphi_{i},
$$

где $b_{\ell i}(J)=b_{i \ell}(J)$.

$2 \mathrm{~b})$ Уравнение $d \omega=0$ для вьведенной 2-формы $\omega$ (3.11) эквивалентно системе $k+1$ уравнений

$$
\mathrm{d}\left(a_{\ell i}(J) \mathrm{d} J_{\ell} \wedge \mathrm{d} J_{i}\right)=0, \quad \mathrm{~d}\left(b_{\ell i}(J) \mathrm{d} J_{\ell}\right)=0, \quad i=1, \ldots, k .
$$

Применяя лемму Пуанкаре, мы получаем

$$
a_{\ell i}(J) \mathrm{d} J_{\ell} \wedge \mathrm{d} J_{i}=\mathrm{d}\left(f_{i}(J) \mathrm{d} J_{i}\right), \quad b_{\ell i}(J) \mathrm{d} J_{\ell}=\mathrm{d} B_{i}(J) .
$$

Последние $k$ уравнений $(i=1, \ldots, k)$ и уравнения $b_{\ell i}(J)=b_{i \ell}(J)$ влекут

$$
b_{\ell i}(J)=b_{i \ell}(J)=\frac{\partial B_{i}(J)}{\partial J_{\ell}}=\frac{\partial B_{\ell}(J)}{\partial J_{i}} .
$$

Поэтому сушествует такая гладкая функция $B(J)$, что

$$
B_{i}(J)=\frac{\partial B(J)}{\partial J_{i}}, \quad b_{\ell i}(J) \mathrm{d} J_{\ell}=\mathrm{d} \frac{\partial B(J)}{\partial J_{i}} .
$$

Таким образом, мы доказали, что любая $f$-инвариантная замкнутая 2-форма $\omega$ имеет вид

$$
\omega=\mathrm{d} \frac{\partial B(J)}{\partial J_{i}} \wedge \mathrm{d} J_{i}+\mathrm{d} f_{i}(J) \wedge \mathrm{d} J_{i}
$$

где $B(J)$ и $f_{i}(J)$ - произвольные гладкие функции.

Формулы (3.7), (3.12) доказьвают, что когомологические классы в группе $H_{B}^{2}(f, \mathscr{O})$ определены классами функций $B(J)$ по модулю линейных функций $c_{1} J_{1}+\cdots+c_{k} J_{k}$. Поэтому группа когомологий $H_{B}^{2}(f, \mathscr{O})$ является бесконечномерной.

3а) Уравнение инвариантности $f^{*} \omega_{3}=\omega_{3}$ для дифференциальной 3-формы

$$
\begin{aligned}
\omega_{3}= & b_{i \ell m}(J, \varphi) \mathrm{d} J_{i} \wedge \mathrm{d} J_{\ell} \wedge \mathrm{d} \varphi_{m}+c_{i \ell m}(J, \varphi) \mathrm{d} J_{i} \wedge \mathrm{d} J_{\ell} \wedge \mathrm{d} J_{m} \\
& +g_{i \ell m}(J, \varphi) \mathrm{d} J_{i} \wedge \mathrm{d} \varphi_{\ell} \wedge \mathrm{d} \varphi_{m}+h_{i \ell m}(J, \varphi) \mathrm{d} \varphi_{i} \wedge \mathrm{d} \varphi_{\ell} \wedge \mathrm{d} \varphi_{m}
\end{aligned}
$$

эквивалентно системе уравнений

$$
\begin{aligned}
h_{i \ell m}(J, \varphi)= & h_{i \ell m}(J, \varphi+J), \\
g_{i \ell m}(J, \varphi)= & g_{i \ell m}(J, \varphi+J)+3 h_{i \ell m}(J, \varphi+J), \\
b_{i \ell m}(J, \varphi)= & b_{i \ell m}(J, \varphi+J)+3 h_{i \ell m}(J, \varphi+J) \\
& +g_{i \ell m}(J, \varphi+J)-g_{\ell i m}(J, \varphi+J), \\
c_{i \ell m}(J, \varphi)= & c_{i \ell m}(J, \varphi+J)+g_{i \ell m}(J, \varphi+J)+h_{i \ell m}(J, \varphi+J) \\
& +\frac{1}{3}\left(b_{i \ell m}+b_{\ell m i}+b_{m i \ell}\right)(J, \varphi+J) .
\end{aligned}
$$


Первые три уравнения (3.14) имеют ту же структуру, что и уравнения (3.8)-(3.10). Поэтому, применяя тот же метод, мы получаем

$$
h_{i \ell m}(J, \varphi)=0, \quad g_{i \ell m}(J, \varphi)=g_{i \ell m}(J), \quad b_{i \ell m}(J, \varphi)=b_{i \ell m}(J),
$$

и соотношения симметрии $g_{i \ell m}(J)=g_{\ell i m}(J)$. Функции $g_{i \ell m}(J)$ по их определению (3.13) удовлетворяют соотношениям $g_{i \ell m}(J)=-g_{i m \ell}(J)$. Легко проверить, что эти два множества соотношений симметрии влекут $g_{i \ell m}(J)=0$. Поэтому последнее уравнение (3.14) принимает вид

$$
c_{i \ell m}(J, \varphi)=c_{i \ell m}(J, \varphi+J)+\frac{1}{3}\left(b_{i \ell m}+b_{\ell m i}+b_{m i \ell}\right)(J) .
$$

Итерируя уравнение (3.15) $N$ раз, мы приходим к уравнению

$$
c_{i \ell m}(J, \varphi)=c_{i \ell m}(J, \varphi+N J)+\frac{N}{3}\left(b_{i \ell m}+b_{\ell m i}+b_{m i \ell}\right)(J) .
$$

Это уравнение имеет тот же вид, что и уравнение (3.6). Поэтому те же аргументы доказывают равенства

$$
c_{i \ell m}(J, \varphi)=c_{i \ell m}(J), \quad b_{i \ell m}(J)+b_{\ell m i}(J)+b_{m i \ell}(J)=0 .
$$

Таким образом, мы доказали, что любая $f$-инвариантная 3-форма $\omega_{3}$ имеет вид

$$
\omega_{3}=b_{i \ell m}(J) \mathrm{d} J_{i} \wedge \mathrm{d} J_{\ell} \wedge \mathrm{d} \varphi_{m}+c_{i \ell m}(J) \mathrm{d} J_{i} \wedge \mathrm{d} J_{\ell} \wedge \mathrm{d} J_{m}
$$

где коэффициенты $b_{i \ell m}(J)$ удовлетворяют уравнениям (3.16).

$3 \mathrm{~b})$ Уравнение $d \omega_{3}=0$ для 3-формы (3.17) эквивалентно системе $k+1$ уравнений

$$
\mathrm{d}\left(b_{i \ell m}(J) \mathrm{d} J_{i} \wedge \mathrm{d} J_{\ell}\right)=0, \quad \mathrm{~d}\left(c_{i \ell m}(J) \mathrm{d} J_{i} \wedge \mathrm{d} J_{\ell} \wedge \mathrm{d} J_{m}\right)=0
$$

где $m=1, \ldots, k$. Применяя лемму Пуанкаре к уравнениям (3.18), мы получаем, что 3-форма $\omega_{3}$ имеет вид

$$
\omega_{3}=\mathrm{d}\left(b_{\ell m}(J) \mathrm{d} J_{\ell}\right) \wedge \mathrm{d} \varphi_{m}+\mathrm{d}\left(a_{i \ell}(J) \mathrm{d} J_{i} \wedge \mathrm{d} J_{\ell}\right) .
$$

Применяя уравнение инвариантности $f^{*} \omega_{3}=\omega_{3}$ к 3 -форме $(3.19)$, мы приходим к уравнению

$$
\mathrm{d}\left(b_{\ell m}(J) \mathrm{d} J_{\ell} \wedge \mathrm{d} J_{m}\right)=0 .
$$

Поэтому лемма Пуанкаре влечет

$$
b_{\ell m}(J) \mathrm{d} J_{\ell} \wedge \mathrm{d} J_{m}=\mathrm{d}\left(B_{m}(J) \mathrm{d} J_{m}\right)
$$

где $B_{m}(J)$ - некоторые гладкие функции. Это уравнение означает, что

$$
b_{\ell m}(J)=\frac{1}{2}\left(\frac{\partial B_{m}(J)}{\partial J_{\ell}}-\frac{\partial B_{\ell}(J)}{\partial J_{m}}\right)+\bar{b}_{\ell m}(J), \quad \bar{b}_{\ell m}(J)=\bar{b}_{m \ell}(J) .
$$

Отсюда мы получаем

$$
\begin{gathered}
\mathrm{d}\left(b_{\ell m}(J) \mathrm{d} J_{\ell}\right)=\mathrm{d}\left(\tilde{b}_{\ell m}(J) \mathrm{d} J_{\ell}\right) \\
\tilde{b}_{\ell m}(J)=\bar{b}_{\ell m}(J)-\frac{1}{2}\left(\frac{\partial B_{m}(J)}{\partial J_{\ell}}+\frac{\partial B_{\ell}(J)}{\partial J_{m}}\right), \quad \tilde{b}_{\ell m}(J)=\tilde{b}_{m \ell}(J) .
\end{gathered}
$$


Уравнения (3.19) и (3.20) эквивалентны равенству $\omega_{3}=d \omega_{2}$, где 2-форма $\omega_{2}$ имеет вид

$$
\omega_{2}=a_{i \ell}(J) \mathrm{d} J_{i} \wedge \mathrm{d} J_{\ell}+\tilde{b}_{\ell m}(J) \mathrm{d} J_{\ell} \wedge \mathrm{d} \varphi_{m}
$$

Вследствие уравнения (3.11) 2-форма $\omega_{2}$ (3.21) является $f$-инвариантной. Поэтому равенство $\omega_{3}=d \omega_{2}$ доказывает, что группа когомологий $H_{B}^{3}(f, \mathscr{O})$ равна нулю.

4) Пусть $\omega_{i}-f$-инвариантные замкнутые 2-формы (3.12), соответствуюшие некоторым гладким функциям $B_{i}(J)$. Дифференциальная 4-форма

$$
\omega_{4}=\sum_{i, j=1}^{N} c_{i j} \omega_{i} \wedge \omega_{j}
$$

является $f$-инвариантной и замкнутой при производных постоянных $c_{i j}$. Формула (3.17) для произвольной $f$-инвариантной 3 -фором $\omega_{3}$ доказывает, что равенство $\omega_{4}=d \omega_{3}$ невозможно, если функции $B_{i}(J)$ в уравнении $(3.12)$ и постоянные $c_{i j}$ находятся в общем положении. Поэтому группа когомологий $H_{B}^{4}(f, \mathscr{O})$ является бесконечномерной.

ЗАмЕчАнИЕ 5. Применяя методы данной работы, можно доказать, что для общих твист-отображений $f(3.1)$, невырожденных в смысле (3.2), все четные группы когомологий $H_{B}^{2 \ell}(f, \mathscr{O})$ являются бесконечномерными при $1 \leqslant \ell \leqslant k$, а все нечетные групшы когомологий $H_{B}^{2 \ell+1}(f, \mathscr{O})$ являются нулевыми. Доказательство будет опубликовано в другой работе.

\section{§ 4. Инвариантный смысл условия невырожденности}

Условие невырожденности (3.2) аналогично условию невырожденности Пуанкаpe [14] для интегрируемых гамильтоновых систем. В работах [6], [7] мы установили инвариантный смысл условия Пуанкаре и условия изоэнергетической невырожденности по Пуанкаре. Для выяснения инвариантного смысла условия (3.2), мы вводим следующее

ОПРЕДЕЛЕНИЕ 1. Пусть $f: M^{n} \rightarrow M^{n}$ - некоторьй диффеоморфизм. Алгебра Ли $f$-инвариантных векторных полей на $M^{n}$ называется алгеброй Ли $\mathscr{S}_{f}$ симметрий диффеоморфизма $f$.

Теорема 3. Твист-отображение $f$ (3.1) тогда и только тогда удовлетворяет условию невырожсдености (3.2) в плотной открытой области $\mathscr{D} \subset B_{a}$, когда его алгебра Ли симметрий $\mathscr{S}_{f}$ абелева.

ДокаЗАТЕльство. Твист-отображение (3.1) является сдвигом на единицу времени вдоль траекторий динамической системы $V$ :

$$
\dot{I}_{1}=0, \ldots, \dot{I}_{k}=0, \quad \dot{\varphi}_{1}=\alpha_{1}(I), \ldots, \dot{\varphi}_{k}=\alpha_{k}(I) .
$$

Поэтому любая симметрия системы (4.1) является симметрией диффеоморфизма (3.1). Следовательно, алгебра Ли $\mathscr{S}_{V}$ симметрий системы (4.1) является подалгеброй Ли в $\mathscr{S}_{f}$. Условие невырожденности (3.2) совпадает с аналогичным условием для динамической системы (4.1), см. уравнение (2.11) работы [6].

Если алгебра Ли $\mathscr{S}_{f}$ абелева, то ее подалгебра Ли $\mathscr{S}_{V}$ также абелева. Поэтому, применяя предложение 1 работы [6], мы получаем, что условие (3.2) выполнено. 
Обратно, если условие невырожденности (3.2) вьполнено, то твист-отображение (3.1) имеет вид $f\left(J_{i}, \varphi_{i}\right)=\left(J_{i}, \varphi_{i}+J_{i}\right)$ в новых координатах $J_{\ell}=\alpha_{\ell}(I)$. Любое векторное поле $U$ в тороидальной области $\mathscr{O}$ имеет вид

$$
U=\sum_{i=1}^{k}\left(U^{i}(J, \varphi) \frac{\partial}{\partial J_{i}}+U^{k+i}(J, \varphi) \frac{\partial}{\partial \varphi_{i}}\right) .
$$

Условие $f$-инвариантности векторного поля (4.2) принимает вид

$$
U^{i}(J, \varphi+J)=U^{i}(J, \varphi), \quad U^{k+i}(J, \varphi+J)=U^{k+i}(J, \varphi)+U^{i}(J, \varphi) .
$$

Эти уравнения имеют тот же вид, что и уравнения (3.3), (3.4). Поэтому, применяя методы теоремы 2 , мы получаем $U^{i}(J, \varphi)=0, U^{k+i}(J, \varphi)=U^{k+i}(J)$. Таким образом, любое $f$-инвариантное векторное поле $(4.2)$ имеет вид

$$
U=\sum_{i=1}^{k} U^{k+i}(J) \frac{\partial}{\partial \varphi_{i}} .
$$

Следовательно, алгебра Ли симметрий $\mathscr{S}_{f}$ абелева.

\section{СПИСОК ЛИТЕРАТУРЫ}

[1] Abraham R., Marsden J. E. Foundations of Mechanics. Reading, Massachusetts: The Benjamin/Cummings Publ. Comp., 1978.

[2] А рнольд В.И. Математические методы классической механики. М.: Наука, 1974.

[3] Bogoyavlenskij O. I. A cohomology of dynamical systems // C. R. Math. Rep. Acad. Sci. Canada. 1995. V. 17. №6. P. 253-258.

[4] Bogoyavlenskij O.I. Theory of tensor invariants of integrable Hamiltonian systems. I. Incompatible Poisson structures // Comm. Math. Phys. 1996. V. 180. № 3. P. 529-586.

[5] Bogoyavlenskij O. I. The $A-B$ - $C$-cohomologies for dynamical systems // C. R. Math. Rep. Acad. Sci. Canada. 1996. V. 18. № 5. P. 199-204.

[6] Bogoyavlenskij O. I. Theory of tensor invariants of integrable Hamiltonian systems. II. Theorem on symmetries and its applications // Comm. Math. Phys. 1997. V. 184. № 2 . P. 301-365.

[7] Bogoyavlenskij O. I. Conformal symmetries of dynamical systems and Poincaré's 1892 concept of iso-energetic non-degeneracy // C. R. Acad. Sci. Paris Sér. I Math. 1998. V. 326. P. 213-218.

[8] De Pham G. Sur l'Analysis situs des varietes a $n$ dimensions // J. Math. Pures Appl. (9). 1931. V. 10. P. 115-200.

[9] Halmos P. R. Lectures on ergodic theory. New York: Chelsea Publ. Comp., 1956.

[10] Hilbert D. Mathematische probleme // Lecture presented at the Second Internat. Congr. Paris. 1900; // Nachr. Akad. Wiss. Göttingen Math.-Phys. Kl. 1900. P. 253-297.

[11] Katok A., Hasselblatt B. Introduction to the modern theory of dynamical systems. Cambridge: Cambridge Univ. Press, 1995.

[12] Колмогоров А. Н. О сохранении условно периодических движений при малых возмущениях функции Гамильтона // Докл. АН СССР. 1954. Т. 98. С. 527-530.

[13] Moser J. On invariant curves of area-preserving mappings of an annulus // Nachr. Akad. Wiss. Göttingen Math.-Phys. Kl. II. 1962. V. 1. P. 1-20.

[14] Poincaré H. Les méthods nouvelles de la mécanique céleste. Paris: Gauthier-Villars et Fils, 1892. 